\title{
OFFICERS OF THE DIVISIONS
}

\author{
Chiltern and Thames Valley Division \\ Chairman: Dr J. Steinert \\ Secretary: Dr Diana Dickens \\ Representative Fellow on Council: Dr E. Shepherd \\ Representative Member on Councii: Dr Diana Dickens \\ Executive Committee: Drs B. Alapin, T. Crow, R. Cundall, \\ H. Dickinson, C. Gore, W. Knapman, J. Knox, E. \\ Shepherd. B. Wharton, V. Wilkinson.
}

East Anglian Division

Chairman: Professor Sir Martin Roth

Secretary: Dr A. Kaeser

Representative Fellow on Council: Dr S. I. Cohen

Representative Member on Council: Dr B. F. Whitehead

Executive Committee: Drs R. Gardner, G. Goldberg, D. Hall, R. Little, D. Lynch, A Mitchell, E. Moran, V. Pillai, F. Sevastianpillai, M. S. Shooter.

Chairman: Dr J. J. Fennelly

Irish Division

Secretary: Dr K. Sinanan

Representative Fellows on Council: Drs A. S. Clenaghan and J. J. Fennelly

Representative Members on Council: Drs D. Dunne and J. Walsh

Executive Committee: Professor I. Browne, Drs P. A. Carney, M. Collins, J. Cooney, P. Cullen, Professor R. J. Daly, Drs L. Daly, D. Dunne, H. Egan, R. Galloway, A. S. Clenaghan, J. Ledwith, W. MacCallum. D. McAlister, P. D. McCarthy, S. D. McGrath, P. McQuaid, P. J. Meehan, G. E. Mullett, U. O'Connell, B. Perry, B. Scally, J. Walsh, D. Walshe, Professor M. Walshe, Dr M. Webb. Co-opted: Drs R. J. Byrne, M. J. Clarke (Inceptor), H. McKee (Trainee) A. Montgomery (Inceptor).

\section{Midland Division}

Chairman: Dr T. L. Dunn

Secretary: Dr E. B. Gordon

Representative Fellow on Council: Dr T. L. Dunn Representative Member on Council: Dr E. B. Gordon Executive Committee: Drs M. Aveline, R. S. Bluglass, I. G. Bronks, B. H. Fookes, J. Harrington, R. Kerry, J. Olive, R. L. Palmer, Sally Pidd, L. Scarth, A. Sims, L. Tarlo.

\section{North East Division}

Chairman: Dr J. M. Roberts

Secretary: Dr D. A. Stephens

Representative Fellow on Council: Dr J. M. Roberts

Representative Member on Council: Dr T. E. Nelson
Executive Committee: Drs J. Blackburn, G. Blessed, P. Cannon, I. Card, N. E. Crumpton, H. de B. Warren G. A. Dransfield, D. L. F. Dunleavy, H. Edgell, J. N. Haworth, Professor I Kolvin, Dr P. Victoria Spencer.

Co-opted: Dr. T. E. Nelson.

\section{North West Division}

Chairman: Dr M. D. Enoch

Secretary: Dr I. Cookson

Representative Fellow on Council: Dr M. Cashman

Representative Member on Council: Dr H. Eaton

Executive Committee: Drs M. Davenport, J. Higgins, S. A. Leslie, S. Lomax, W. P. Maragakis, G. N. Mathur, M. Silverman, A. Theodossiadis, S. Wright. Co-opted: Drs M. W. Forth, A. W. Johnson, Susanne Shafar.

\section{Scottish Division}

Chairman: Dr G. C. Timbury

Secretary: Dr W. D. Boyd

Representative Fellows on Council: Drs W. D. Boyd and A. B. Sclare

Representative Members on Council: Drs J. Evans and M. R. Malcolm

Executive Committee: Drs E. A. Ballinger, J. K. Binns, R. Davidson, Professor R. E. Kendell, Drs $M$. O'Gorman, M. Rennie, and the Chairman or Secretary of each of the Specialist Sections and Groups in the Division.

\section{Southern Division}

Chairman: Dr R. Maggs

Secretary: Dr K. Hamadah

Representative Fellow on Council: Dr. E. D. West

Representative Member on Council: Dr A. C. Smith

Executive Committee: Drs A. A. Black, O. Briscoe, J. Cleobury, K. Fraser, M. Markowe, B. Matthews, J. Merry, K. O'Keefe, E. S. Paykel, Daphne Smedberg, J. S. Stead, R. Veall, P. Woolf.

\section{Chairman: Dr D. F. Early \\ Secretary: Dr C. Davies}

South West Division

Representative Fellow on Council: Dr D. F. Early Representative Member on Council: Dr C. Davies Executive Committee: Drs H. J. Crow, I. G. Christie, D. H. Dick, M. J. Gay, A. L. Horton, J. Jancar, G. E. Langley, J. S. Lyon, H. G. Morgan, P. N. Nott, G. D. P. Wallen, A. E. Wilson. 


\title{
Welsh Division
}

Chairman: Dr J. M. Cuthill Secretary: Dr A. M. P. Kellam Representative Fellow on Council: Dr T. Gwynne Williams Representative Member on Council: $\operatorname{Dr}$ A. M. P. Kellam Executive Committee: Drs K. G. Mohan Bhakta, M. J. Craft, T. J. G. Davies, M. Harper, J. M. Hughes, P.
Jackson, D. C. Jones, E. M. E. Jones, Elinor Kapp, A. B. Rolfe, R. Viswanathan, R. J. W. Williams, T. Gwynne Williams.

Ex-officio: Drs M. W. Annear, H. Rosenburg, T. T. Westhead, Professor K. Rawnsley.

\section{OFFICERS OF SECTIONS AND GROUPS}

\section{Sections}

Child and Adolescent Psychiatry Section

Chairman: Dr W. Lumsden Walker

Secretary: Dr H. C. Cameron

Representative Fellow on Council: Dr W. Lumsden Walker Representative Member on Council: Dr H. C. Cameron

Executive Committee: Drs I. S. Berg, Dora Black, J. M. Black, P. Bruggen, C. L. Casimir, J. Evans, Eva A. Frommer, Ann Gath, Professor I. Kolvin, Drs M. Lindsay, W. Parry-Jones, A. R. Nicol, P. Pinkerton, D. G. Melville Thomas, the Chairmen and Secretaries of the Section in Ireland, Scotland and Wales.

Co-opted: Drs Anne Bolton, M. Best, Professor P. Graham, Dr W. Warren.

Observers: Drs L. Brierley, G. Kerr.

Chairman: Dr R. Bluglass

Forensic Psychiatry Section

Secretary: Dr P. Bowden

Representative Fellow on Council: Dr P. T. d'Orban

Representative Member: $\mathrm{Dr} \mathrm{J}$. Hamilton

Executive Committee: Drs A. Campbell, B. D. Cooper, M. Ellis, Professors T. C. N. Gibbens, J. Gunn, Drs H. Hunter, J. MacKeith, A. McQuaid, H. Milne, I. Pierce James, D. G. A. Westbury, P. G. Woolf. Co-opted: Drs J. Higgins, D. E. McDonald, V. Molony, R. C. Ingrey-Sinn, D. O. Topp. Observer: Dr L. J. Warnants.

Chairman: Dr Valerie Cowie

Mental Deficiency Section

Secretary: Dr W. R. McKibben

Representative Fellow on Council: Dr Valerie Cowie Representative Member on Council: Dr W. R. McKibben

Executive Committee: Drs J. T. Bavin, J. A. Corbett, M. Craft, A. S. R. Goonetellike, J. A. Hatrick, J. Jancar, M. Leyshon, D. M. Lynch, N. Quinn, G. E. Roberts, P. Sylvester, R. M. Veall. Co-opted: Drs F. Broderick, W. I. Fraser, Y. Khan, D. A. Primrose, E. W. Shepherd, H. Smyth. Observer: Dr R. Wilkins.
Section for the Psychiatry of Old Age

Chairman: Dr R. A. Robinson

Secretary: Dr P. M. Jeffreys

Representative Fellow on Council: Dr R. A. Robinson

Representative Member on Council: Dr P. M. Jeffreys

Executive Committee: Awaiting election

\section{Psychotherapy Section}

Chairman: Dr I. S. Kreeger

Secretary: Dr D. C. James

Representative Fellow on Council: Dr I. S. Kreeger

Representative Member on Council: Dr J. R. Pedder

Executive Committee: Drs M. Aveline, J. Cobb, A. Cook-

lin, J. Denford, M. Feldman, M. Glasser, R. H.

Gosling, J. R. Pedder, B. J. Muir, J. Steiner, J. D. Templeton, $\mathrm{H}$. Woolf.

Co-opted: Drs J. Evans, J. C. Lucas, H. Rosenberg, D. J. Ward, D. Will.

Observer: Dr L. Brierley.

V. $\quad$ Secri

Secretary: Dr R. Murray

Executive Committee: Awaiting election

\author{
Social and Community Psychiatry Group \\ Chairman: Dr J. P. Leff \\ Secretary: Dr A. C. Brown \\ Executive Committee: Drs D. H. Bennett, A. Brook, \\ Professor J. E. Cooper, Drs J. Corbett, R. de \\ Alarcon, J. D. W. Fisher, Professor S. Hirsch, Drs P. \\ M. Jefferys, Josephine Lomax-Simpson, C. Parkes, \\ R. Rosser, A. Ryle, U. P. Seidel, Professor J. K. \\ Wing. \\ Co-opted: Drs A. Golding, H. Mair, Sheila Mann, \\ J. Schlicht. \\ Observer: Dr P. Mason.
}

